\title{
Assessment of technical and financial benefits of $A C$ and DC microgrids based on solar photovoltaic
}

\author{
Shahid Ullah ${ }^{1} \cdot$ Ahmed M. A. Haidar ${ }^{1,2} \cdot$ Hushairi Zen ${ }^{1}$
}

Received: 28 May 2019 / Accepted: 10 February 2020

(c) Springer-Verlag GmbH Germany, part of Springer Nature 2020

\begin{abstract}
Microgrid deployments are expanding around the world as the most suitable solution to integrate distributed renewable energy sources to meet the increasing load demands and to power-up the remote areas. The installation of DC microgrid can improve system efficiency and reduces the cost of electrical infrastructure compared to the AC microgrid. However, the main challenge of implementing DC microgrid is the existing structure of the AC distribution system. In addition to the previous researches performed on DC microgrids, this paper proposes a framework to assess the technical and financial benefits of implementing the AC and DC microgrids. The power loss, voltage drop and system efficiency have been investigated for the AC and DC microgrids during the steady-state condition. Furthermore, the dynamic behaviors of AC and DC microgrids have been analyzed when each system subjected to disturbance such as short-circuit fault, aiming to evaluate the system response. In the next stage, techno-economic analysis has been carried out to determine the optimal size of solar PV system connected to each AC or DC microgrid with its energy storage, according to the meteorological and load profile data of the selected remote area in Sarawak (Malaysia). The study presented in this paper justifies that DC microgrid is potentially more beneficial than AC microgrid. However, the stability of the system during fault condition is the main problem in the DC microgrid. Therefore, it can be concluded that the protection and control of DC microgrids should be the key areas of future researches.
\end{abstract}

Keywords AC and DC microgrid configurations · Techno-economic analysis · Cost optimization $\cdot$ System losses

\section{Introduction}

According to the statistics of the International Energy Agency, nearly 1.3 billion people around the world have no access to electricity, especially in rural and remote areas where utility power grid extension may be challenging as well as uneconomical due to lower energy demands, thereby, defeating the goals of sustainable development [1]. It is expected that the global energy demand may grow by nearly $40 \%$ of that in 2015 , and thus, a mindset has been developed to focus on power generation from distributed renewable energy sources (DRES) to limit the dependency on fossil fuels. About 169 countries adopted policies that support renewable energy technologies, approximately $6.5 \%$ annual

Shahid Ullah

16010178@siswa.unimas.my

1 Department of Electrical and Electronic Engineering, Universiti Malaysia Sarawak, Kota Samarahan, Malaysia

2 School of Mechanical and Electrical Engineering, University of Southern Queensland, Toowoomba, Australia growth in renewable energy production is expected over the next 20 years and the share of renewable generation in primary energy production is likely to grow from $3 \%$ in 2015 to $8 \%$ by the end of 2035 . This is not only because of the limitations of fossil fuels but also to rein in electricity costs, replace aging infrastructure, minimize greenhouse emissions, deforestation and provide reliable electricity to remote areas [2-4]. In 2018, a total capacity of 181 gigawatts (GW) of renewable power was added and the total global renewable power capacity grew to $2378 \mathrm{GW}$, providing more than $26 \%$ of global electricity generation by year's end. The highest growth was experienced in solar energy as almost $100 \mathrm{GW}$ of solar PV systems were installed (accounting for 55\% of renewable capacity additions in 2018). This is due to the fact that solar energy is the most prominent among renewable energy sources because of its simplicity and flexible power production capability (from milliwatts to Gigawatts) [5].

Although utility grid propagation or extension is the first option for electrification and should be economical when compared to other off-grid options, however, it is a challenge to integrate DRES such as PV systems, wind turbines, 